\title{
Neuroserpin Protects Rat Neurons and Microglia-Mediated Inflammatory Response Against Oxygen-Glucose Deprivation- and Reoxygenation Treatments in an In Vitro Study
}

\author{
Xuelian Yang a,c Tetsuya Asakawa ${ }^{\mathrm{d}, \mathrm{e}}$ Sha Han ${ }^{\mathrm{a}}$ Ling Liu ${ }^{\mathrm{a}}$ Wei Lia, ${ }^{\mathrm{a}}$ Weiwen Wu $\mathrm{W}^{\mathrm{a}, \mathrm{g}}$ \\ Yunhe Luo ${ }^{a}$ Wenjie Cao ${ }^{a}$ Xin Cheng ${ }^{a}$ Baoguo Xiao ${ }^{b}$ Hiroki Nambad \\ Chuanzhen Lu ${ }^{a, b}$ Qiang Dong ${ }^{a, b}$ Liang Wang ${ }^{a, b}$ \\ aDepartment of Neurology, Huashan Hospital, Fudan University, Shanghai, China; 'DInstitute of \\ Neurology, Huashan Hospital, Fudan University, Shanghai, China; 'Department of Neurology, Shanghai \\ Pudong New Area Gongli Hospital, Shanghai, China; dDepartment of Neurosurgery, Hamamatsu \\ University School of Medicine, Hamamatsu, Japan; eDepartment of Psychiatry, Hamamatsu University \\ School of Medicine, Hamamatsu, Japan; fDepartment of Neurology, Zhongshan Hospital, Fudan \\ University, Shanghai, China; 9Department of Neurology, Qingpu Hospital, Fudan University, Shanghai, \\ China
}

\section{Key Words}

Neuroserpin • Oxygen-glucose deprivation $・$ Neuron $・$ Microglia $・$ Neuroprotection

\begin{abstract}
Background/Aims: Neuroserpin (NSP) is known for its neuroprotective role in cerebral ischemic animal models and patients. Our laboratory conducted a series of investigations on the neuroprotection of NSP in different cells in the brain. In the present study, we further observe the effects of NSP on neurons and microglia-mediated inflammatory response following oxygen-glucose deprivation (OGD), and explore possible mechanisms related to neuroprotection of OGD in the central nervous system (CNS). Methods: Neurons and microglia from neonatal rats were treated with OGD followed by reoxygenation (OGD/R). To confirm the effects of NSP, the neuronal survival, neuronal apoptosis, and lactate dehydrogenase $(\mathrm{LDH})$ release were measured in cultured neurons. Furthermore, the levels of IL-1 $\beta$ and nitric oxide (NO) release were also detected in cultured microglia. The possible mechanisms for the neuroprotective effect of NSP were explored using Western blot analysis. Results: NSP administration can reverse abnormal variations in neurons and microgliamediated inflammatory response induced by OGD/R processes. The neuronal survival rate, neuronal apoptosis rate, and LDH release were significantly improved by NSP administration in neurons. Simultaneously, the release of IL-1 $\beta$ and NO were significantly reduced by NSP




\section{Cellular Physiology Cell Physiol Biochem 2016;38:1472-1482 \begin{tabular}{l|l} 
and Biochemistry Published online: April 04, 2016 & $\begin{array}{l}\text { Co 2016 S. Karger AG, Basel } \\
\text { www.karger.com/cpb }\end{array}$ \\
\hline
\end{tabular} \\ Yang et al.: NSP Shows Neuroprotection on Neurons and Microglia-Mediated Inflam- matory Response}

in microglia. Western blot showed that the expression of ERK, P38, and JNK was upregulated in microglia by the OGD/R treatment, and these effects were significantly inhibited by NSP. Conclusion: These data verified the neuroprotective effects of NSP on neurons and microgliamediated inflammatory response. Inhibition of the mitogen-activated protein kinase (MAPK) signaling pathways might play a potential role in NSP neuroprotection on microglia-mediated inflammatory response, which needs further verification.

Copyright $@ 2016$ S. Karger AG, Basel

\section{Introduction}

Neuroserpin (NSP) is known to inhibit tPA and plays critical roles in cerebral development, neuronal survival, and synaptic plasticity [1]. The neuroprotective role of NSP has received widespread attention, although these mechanisms have been poorly understood. NSP demonstrates widespread neuroprotective effects not only on stroke but also on neurodegenerative disorders [2-5] in both patients [6] and animal models [1, 7, 8]. Recently, the neuroprotective role of NSP on ischemic/hypoxia cerebral cells has been increasingly investigated. As early as 1999, Docaqne et al. found that NSP was expressed in both neurons and astrocytes [9]. Rodriguez-Gonzalez et al. reported that oxygen-glucose deprivation (OGD) processes induced morphological changes and increased cell death in mixed cortical cultures containing both astrocytes and neurons, whereas NSP administration could reverse these changes [10]. Our laboratory also conducted a series of studies on different cells that comprise the central nervous system (CNS). Our previous study verified the neuroprotective effects of NSP on astrocytes. We found that OGD followed by reoxygenation (OGD/R) reduced the astrocyte survival rate, enhanced release of relative lactate dehydrogenase (LDH), nitric oxide (NO), and TNF- $\alpha$. However, NSP administration might reverse these variations, namely by reducing the release of LDH, NO, and TNF- $\alpha$ as well as by enhancing the survival rate of astrocytes. We found that the expression of $\mathrm{p}-\mathrm{IKKB} \alpha / \beta$ and $\mathrm{P} 65$ were upregulated by the OGD/R processes, and these effects were significantly reversed by NSP administration. The NSP-induced amelioration could be significantly reversed by the administration of sc3060, an NF- $\kappa B$ pathway inhibitor. Thus, we concluded that the inhibition of NF- $\kappa B$ signaling pathways may be a potential mechanism in the protective effects of NSP on astrocytes [11]. Neurons and microglia are the other important components in the brain. It is interesting to verify whether NSP exerts neuroprotective effects on neurons and microglia-mediated inflammatory response.

Neurons, also known as nerve cells, are the core components of the CNS. Neurons can form a neural network through dendrites and axons, which are electrically excitable. Neurons along with the neural network are the basis for neurological functions [12]. In this regard, the neuroprotection of injured neurons may improve brain functions, which is beneficial for several neurological diseases. Microglia are known as the resident macrophages of CNS, which play a crucial role in active immune defense mechanism in the CNS. Microglia are a type of glial cell different from astrocytes. They are lesser in number than astrocytes [13] but are also distributed over a larger range in the CNS [14]. Traditionally, microglia have been regarded as "scavenger cells," which can eliminate damaged neurons, plaques, and other infectious agents. When antibodies or infectious agents cross the blood-brain barrier or are introduced into the CNS directly, microglia must promptly phagocytose them and reduce inflammation to maintain the stability of the CNS. In this regard, microglia are very sensitive to even small stimuli [15]. Therefore, microglia are crucial for maintaining the normal functions of the CNS. Recently, the roles of microglia in chronic neuroinflammation, aging, neurodegeneration, infections, and neuropathic pain are increasingly being emphasized. Because of the crucial roles of neurons and microglia in the CNS, the verification of neuroprotective effects of NSP on neurons and microglia-mediated inflammatory response is very important for clarifying the biological significance of NSP. On the basis of previous 
studies conducted on mixed cells [10] and astrocytes [11] as part of a series of studies in our laboratory, the aim of the present study is to verify the neuroprotective effects of NSP on neurons and microglia-mediated inflammatory response.

It has been reported that besides neurons, NSP linked to the differentiation and activation of the human myeloid lineage [16]. In a murine model of chronic arterial-wall inflammation, NSP treatment has been shown to block vascular inflammatory cell responses and suppress proinflammatory Th1 lymphocyte differentiation [17]. Recently, it was observed that NSP treatment also significantly reduced macrophage infiltration in tumors [18], revealing that NSP may have important regulatory effect on macrophage/microglia. Furthermore, we here attempted to explore potential mechanisms involved in the neuroprotective effect of NSP on microglia-mediated inflammatory response.

\section{Materials and Methods}

\section{Preparation of neurons and microglia}

All cells were acquired from newborn Sprague-Dawley rats (age, $<24 \mathrm{~h}$; Shanghai Institute of the Chinese Academy of Science, China). All rats were carefully treated as per the National Institute of Health Guidelines for the Care and Use of Laboratory Animals. All experimental procedures were approved by the Animal Care and Use Committee of Fudan University (authorization No: 061105308). Rats were deeply anesthetized by the intraperitoneal injections of sodium pentobarbital $(50 \mathrm{mg} / \mathrm{kg}$ body weight); their cortical hemispheres were removed from the skull and meninges.

Primary cortical neuron cultures were prepared as described previously [19, 20]. Briefly, the tissues were dissociated by digestion using $0.125 \%$ trypsin for $10 \mathrm{~min}$ at $37^{\circ} \mathrm{C}$ with gentle trituration. The homogenate was placed in 96 well plates or $100 \mathrm{~mm}$ dishes at a density of 3-10 $\times 10^{5} \mathrm{cell} / \mathrm{mL}$ and maintained at $37^{\circ} \mathrm{C}$ in a humidified $5 \% \mathrm{CO}_{2}$ incubator. Neurons were cultured in Dulbecco's modified Eagle's medium (DMEM; Sigma-Aldrich) containing L-glutamine plus 10\% fetal bovine serum (FBS; Hyclone, Thermo Scientific, Waltham, USA) and $10 \%$ horse serum. This was then replaced with neurobasal, a medium supplemented with 2\% B27 and 0.5 mM L-glutamine every 3-4 days. Cultures were treated with 5-fluoro2-deoxyuridine and uridine on the third day after plating to suppress glial growth. Under these conditions, neuronal purity exceeded $90 \%$, which was evaluated by immunocytochemical staining with antibodies against neurofilament proteins and glial fibrillary acidic proteins. These cells were used for the following in vitro experiments between day 8 and 10 (DIV 8-10).

Glial cells were isolated from the homogenate and were placed in a $75 \mathrm{~cm}^{2}$ flask at a density of $2 \times 10^{5}$ cells $/ \mathrm{mL}$ of DMEM (Sigma-Aldrich) supplemented with $20 \%$ FBS. The flasks were then placed in a humidified atmosphere containing $5 \% \mathrm{CO}_{2}$ and $95 \%$ air at $37^{\circ} \mathrm{C}$. The culture medium was changed every 48-72 h. Microglia cells were isolated by shake from the mixed glial population when mixed glial cells were confluent (12-14 days) [21]. The purity of microglia was confirmed by immunofluorescence labeling using CD11b, a marker of microglia (concentration, $20 \mu \mathrm{g} / \mathrm{mL}$; Abcam, UK).

Processes of oxygen-glucose deprivation followed by reoxygenation (OGD/R)

The OGD/R processes were performed as previously described [11,22, 23], with minor modifications. Briefly, the original glucose-containing media were removed from all treatment groups and kept aside for future use. Neurons or microglia were washed three times with deoxygenated glucose-free extracellular fluid (ECF) containing the following components: $140 \mathrm{mM} \mathrm{NaCl}$, $5.4 \mathrm{KCl}, 25 \mathrm{mM}$ HEPES, $20 \mathrm{mM}$ glucose, $1.3 \mathrm{mM} \mathrm{CaCl}_{2}, 1.0 \mathrm{mM} \mathrm{MgCl}, 0.0005 \mathrm{mM}$ TTX ( $\mathrm{pH} 7.4$ ), and 320-335 mOsm]; then, they were incubated in that fluid at $\mathrm{pH} 7.4$ for OGD in an anaerobic chamber (Model 1025; Forma Scientific) with an atmosphere $\left(<0.1 \%\right.$ of $\mathrm{O}_{2}$ ) containing $85 \% \mathrm{~N}_{2}, 10 \% \mathrm{H}_{2}$, and $5 \% \mathrm{CO}_{2}$ at $37^{\circ} \mathrm{C}$. In this study, $\mathrm{OGD}$ was continued for $1.5 \mathrm{~h}$ for neurons as opposed to a 3-h OGD for microglia. OGD was terminated after 90 min by removing cultures from the chamber, washing the cultures with oxygenated glucose-containing ECF, adding the original media, and incubating the cultures at $37^{\circ} \mathrm{C}$ in a normoxic incubator for $0-24-\mathrm{h} \mathrm{R}$, according to the experimental design. The control cells were washed and incubated in a normoxic incubator throughout the common normoxic astrocyte-culturing medium (blank control). 


\section{Cellular Physiology Cell Physiol Biochem 2016;38:1472-1482

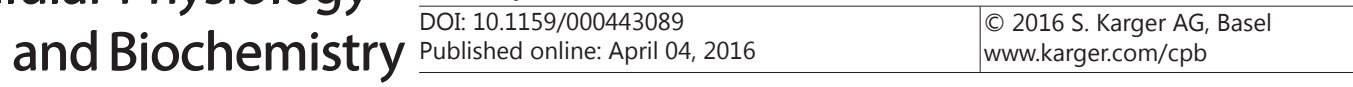 \\ Yang et al.: NSP Shows Neuroprotection on Neurons and Microglia-Mediated Inflam- matory Response}

\section{Administration of NSP}

We adopted a method similar to that conducted in our previous study [11]; briefly, recombinant human NSP (PeproTech, USA) was diluted with MEM containing 10\% FBS at $24 \mathrm{~h}$ before the experiments. NSP $(25 \mu \mathrm{g} / \mathrm{ml})$ was randomly added to the cultured neurons and microglia $(100 \mu \mathrm{L} /$ well $)$ in a 96 -well filter plate (AcroPrep, USA). We subjected NSP solution to OGD treatment for $1.5 \mathrm{~h}$ followed by $4 \mathrm{~h} \mathrm{R}(1.5 \mathrm{~h}$ $\mathrm{OGD} / 4 \mathrm{~h} \mathrm{R}$ ) for neurons, and OGD treatment for $3 \mathrm{~h}$ followed by $24 \mathrm{~h} \mathrm{R}$ (3h OGD/24h R) for microglia.

\section{Immunocytochemical staining for neurons}

Cells were grown on glass coverslips for 8 days and exposed to OGD/R processes (OGD1.5hR4h for neurons, while OGD3hR24h for microglia) or a blank control; then, they were fixed for $10 \mathrm{~min}$ in $0.1 \mathrm{M}$ PBS containing $4 \%$ paraformaldehyde. After washing in PBS, cells were permeabilized for $15 \mathrm{~min}$ in PBS-T $(0.3 \%$ Triton X-100 in PBS), blocked in PBS-T containing 3\% FBS and then incubated with rabbit anti-ASIC1a and mouse anti-MAP2 monoclonal antibodies (Beyotime, China) in PBS-T containing 3\% FBS overnight at $4^{\circ} \mathrm{C}$. After three washes in PBS, cells were incubated for $1 \mathrm{~h}$ with rhodamine-labeled anti-mouse (Beyotime, China) and FITC-labeled anti-rabbit secondary antibodies (Beyotime, China) at room temperature in the dark. Immunostained neuronal cells were then incubated with Hoechst $33342(10 \mu \mathrm{g} / \mathrm{mL}$; Beyotime, China) for $10 \mathrm{~min}$ and washed three times in PBS. Fluorescent labeling was then visualized under a fluorescence microscope (Olympus BX 51, Japan) equipped with adequate filter cubes.

\section{Cell viability assay}

Cell viability was assessed by WST-8 assay using a Cell Counting Kit-8, according to the manufacturer's instructions, which forms colored formazan based on cellular dehydrogenase activity in viable cells. At the indicated time points, $10 \mu \mathrm{L}$ Cell Counting Assay Kit-8 solution was added to each well, and cells were incubated for another $1-2 \mathrm{~h}$ at $37^{\circ} \mathrm{C}$. The results are expressed as the percentage of viable cells relative to untreated controls by absorbance at $450 \mathrm{~nm}$ with a reference wavelength of $630 \mathrm{~nm}$ measured using a microplate reader (Spectra Max M7; Molecular Devices, USA). Each experiment contained eight readings for one experimental condition.

\section{TUNEL staining for the evaluation of neuron apoptosis}

Neuronal apoptosis was evaluated by a method of the terminal deoxynucleotidyl transferase (TdT)mediated dUTP nick end labeling (TUNEL) [24]. Briefly, in the present study, TdT binds to the exposed 3'-OH ends of the DNA fragments generated in response to apoptotic signals, where it catalyzes the addition of biotin-labeled and unlabeled deoxynucleotides. Biotinylated nucleotides are detected using a streptavidinhorseradish peroxidase (HRP) conjugate. The labeled samples were treated with diaminobenzidine (DAB) to generate an insoluble colored substrate at the site of DNA fragmentation. Counterstaining with methyl green aided in the morphological evaluation and characterization of normal and apoptotic cells. Then, cortical cell cultures (DIV 8) were plated on glass coverslips and fixed in 4\% paraformaldehyde in PBS, before being subjected to TUNEL staining using the FragEL ${ }^{\mathrm{TM}}$ DNA Fragmentation Detection Kit (Calbiochem, USA), according to the manufacturer's instructions. The cells were examined under a light microscope (Olympus BX51, Japan). The number of TUNEL-positive (apoptotic) cells that appeared dark brown and the total number of cells that appeared as a mixture of dark brown and bluish-green were determined in eight randomly chosen microscopic fields, each at a magnification of 200x. Data were expressed as the ratio of apoptotic neurons to the total number of neurons.

\section{The measurement of $L D H$ release}

LDH release was measured following a classic method $[11,25]$. Briefly, the medium $(50 \mu \mathrm{L})$ was shifted from the culture well to a 96 well plate and mixed with a $50 \mu \mathrm{L}$ of reaction mixture with a commercial LDH detection kit (Sigma, USA). The mixtures were incubated at $23^{\circ} \mathrm{C}$ in the dark for $30 \mathrm{~min}$; then, $50 \mu \mathrm{L}$ of stop solution was added to each well. After $30 \mathrm{~min}$, the absorbance was read using a microplate reader (492 nm; Thermo, USA). The maximum LDH release was investigated in each well at the end of each experiment following repeated freezing and thawing. Each experiment was performed in triplicates, with each experiment containing eight readings. Results are expressed as a percentage of the maximum LDH release, after subtracting background levels determined from the medium alone.

\section{KARGER}




\section{Cellular Physiology Cell Physiol Biochem 2016;38:1472-1482

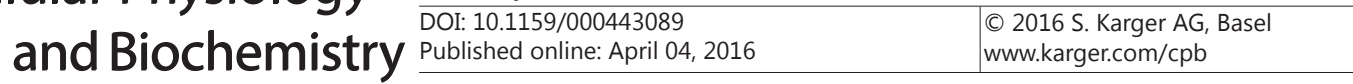 \\ Yang et al.: NSP Shows Neuroprotection on Neurons and Microglia-Mediated Inflam- matory Response}

The measurement of nitric oxide (NO) and $I L-1 \beta$

Enzyme-linked immunosorbent assay (ELISA) was employed to measure the release of NO and IL-1 $\beta$ from microglia by NSP using the NO ELISA kit and IL-1 $\beta$ ELISA kit (ADL, USA), according to the manufacturer's instructions. NO was measured as described in our previous study [11]. In brief, a standard curve was generated to detect concentrations between 1 and $100 \mu \mathrm{M}$ of nitrite per well. Then, $100 \mu \mathrm{L}$ microglia solutions were centrifuged at $10,000 \mathrm{~g}$ at $4^{\circ} \mathrm{C}$ for $2 \mathrm{~min}$, and the clarified supernatant was recovered. Ice-cold (5\%) metaphosphoric acid (Sigma-Aldrich) was mixed with clarified samples in a ratio of $1: 1$ (v:v; mixing and centrifuging at $10,000 \mathrm{~g}$ for $5 \mathrm{~min}$ ). Supernatants along with standards were exposed to nitrate reductase for $1 \mathrm{~h}$ at room temperature $\left(23^{\circ} \mathrm{C}\right)$ to transform nitrate to nitrite. Using the enhancer, we employed Griess reaction reagents to convert nitrite to a purple azo-chromophore compound. Optical density was measured using a microplate reader (Multiscan MK3; Thermo Labsystems, USA) at $450 \mathrm{~nm}$ with a wavelength correction at $570 \mathrm{~nm}$. IL-1 $\beta$ was measured according to the manufacturer's instructions.

\section{Western blot analysis}

A standard Western blot analysis was employed to clarify the potential effect of NSP on microglia MAPK pathway $[26,27]$. We added the NSP into cultured microglia and adjusted the concentration of the NSP to $25 \mu \mathrm{g} / \mathrm{ml}$ for $1 \mathrm{~h}$; the cells were treated with OGD3h followed by $24 \mathrm{~h}$ of reoxygenation (OGD3hR24h). The blank control group was not subjected to the OGD/R treatment. After OGD/R, the cells were subjected to Western blot analysis. As previously described [11], microglia were scraped into lysis buffer A (10 mM HEPES, pH 7.4; 10 mM KCl; 0.1 mM EDTA; 0.1 mM EGTA; 1 mM DTT; 1 mM PMSF; $1 \mu \mathrm{g} / \mathrm{mL}$ aprotinin; and $1 \mu \mathrm{g} / \mathrm{mL}$ leupeptin) and centrifuged at $14,000 \mathrm{~g}$ for $10 \mathrm{~min}$. Supernatants were collected and used as cytosolic fractions, whereas pellets were resuspended in lysis buffer B (20 mM HEPES, pH 7.4; $400 \mathrm{mM} \mathrm{NaCl} ; 1 \mathrm{mM}$ EDTA; 10\% glycerol; 1 mM DTT; 1 mM PMSF; $1 \mu \mathrm{g} / \mathrm{mL}$ aprotinin; and $1 \mu \mathrm{g} / \mathrm{mL}$ leupeptin) and incubated on ice for $30 \mathrm{~min}$. Then, we centrifuged these lysates at 14,000 g for $15 \mathrm{~min}$ and collected supernatants. Protein concentrations in the supernatants were measured using the BCA protein assay kit (Pierce Manufacturing, USA), and samples containing equal amounts of protein (30 $\mu \mathrm{g}$ ) were subjected to electrophoresis on $10 \%$ SDS-polyacrylamide gels. After transfer to nitrocellulose membranes, the membranes were incubated in $4 \%$ milk in PBS for $1 \mathrm{~h}$ at room temperature and then incubated for $12 \mathrm{~h}$ at $4^{\circ} \mathrm{C}$ with antibodies (diluted 1:1000) against ERK1/2 (t-ERK1/2; Kangchen, China), phospho-ERK1/2 (p-ERK1/2; CST Co, USA); P38 (Kangchen, China), phospho-P38 (Kangchen, China), JNK (Kangchen, China), and phospho-JNK (Santa Cruz, USA), flowed by corresponding peroxidase-conjugated anti-rabbit or anti-mouse secondary antibodies (Santa Cruz Biotechnology, USA). The bands were visualized using chemiluminescence reagents provided with the ECL kit (Santa Cruz Biotechnology, USA). Densitometry was performed as the index of quantitative analysis using the Quantity One software (version 4.2.2, BioRad Laboratories, USA).

\section{Data analysis and statistics}

All experiments were repeated at least three times in separate cell preparations and the average data were calculated. Data were recorded as the mean \pm standard error and were analyzed using the SPSS 13.0.0 software (SPSS, USA). Two-way ANOVA was used for comparisons. $\mathrm{P}<0.05$ was considered statistically significantly. All the tests were two-sided.

\section{Results}

\section{The neuroprotective effects of NSP on neurons}

Figure 1 shows the protective effects of NSP on neurons treated by OGD/R. Figure 1A shows the morphological variations of cultured neurons indicated by OGD/R processes; the left column is an image of normal cultured neurons, which are round-shaped with abundant dendrites. The middle column is an image of neurons that had undergone OGD/R processes. The number of neurons reduced and dendrites disappeared, which indicated the neurotoxic effects of OGD/R processes. The right column shows that NSP can protect from neuron injury induced by OCD/R processes.

The survival rate of neurons was significantly reduced after OGD 1.5h/R4h (Fig. 1B, $\mathrm{p}<0.01, \mathrm{OGD} / \mathrm{R}$ group vs. blank control). However, the administration of NSP significantly 


\section{Cellular Physiology Cell Physiol Biochem 2016;38:1472-1482 and Biochemistry

Fig. 1. The neuroprotective effects of NSP on neurons. Primary neurons were cultured and treated with OGD1.5h/R24h or OGD1.5h/R24h + NSP. (A) the morphological variations of neurons, (B) the rate of neuronal survival, (C) the rate of neuronal apoptosis and (D) LDH release. * means $\mathrm{p}<0.05$; ** means $\mathrm{p}<0.01$, the bar $=10 \mu \mathrm{m}$.

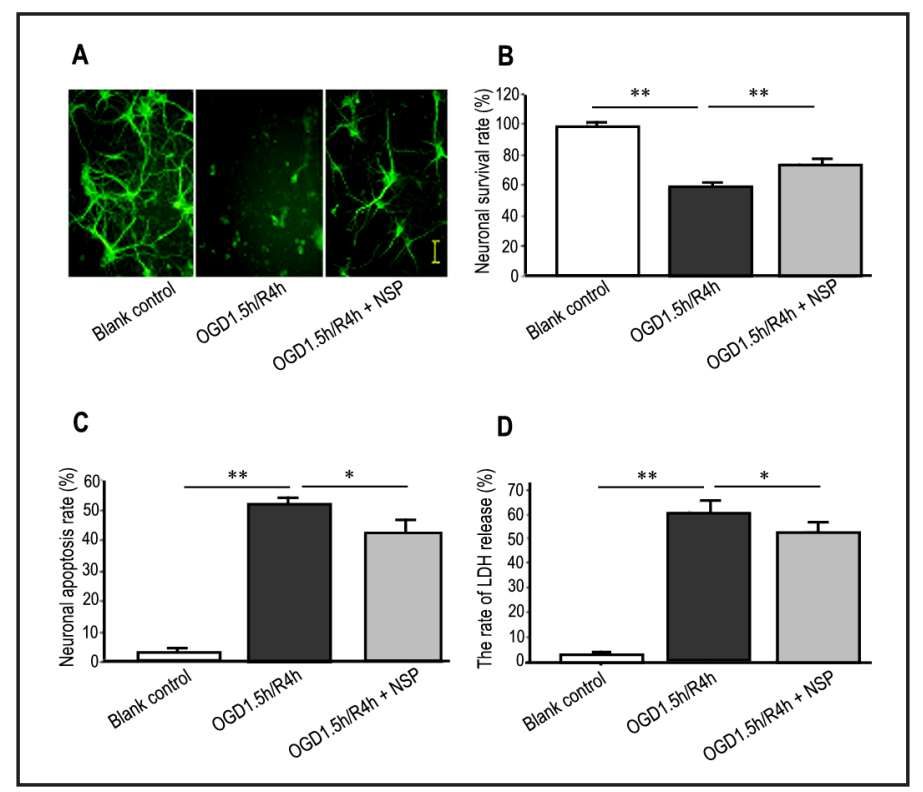

increased the survival rate of neurons (Fig. $1 \mathrm{~B}, \mathrm{p}<0.01$ ). The rate of neuron apoptosis was significantly increased after OGD1.5h/ R4h (Fig. 1C, $p<0.01$, OGD/R group vs. blank control). However, the administration of NSP significantly decreased the rate of neuron apoptosis (Fig. 1C, p < 0.05). The LDH release from neurons dramatically increased after OGD1.5h/R4h (Fig. 1D, p < 0.01, OGD/R group vs. blank control). However, the administration of NSP significantly decreased LDH release (Fig. 1D, p < 0.05). The results demonstrate the neuroprotective effects of NSP in cultured neurons.

The neurotoxic factors from microglia induced by $O G D / R$ processes

Figure 2A shows the morphological changes of microglia induced by $\mathrm{OGD} / \mathrm{R}$ processes. The left column is an image of normal cultured microglia. The shape of a normal microglia is characteristic, namely spindle-like cells with long branches. The right column is an image of microglia that had undergone $\mathrm{OGD} / \mathrm{R}$ treatments. The density of the cells was reduced and the cells became rounded and wrinkled (Fig. 2A). Microglia that had undergone OGD $3 \mathrm{~h}$ exerted significantly higher production of IL-1 $\beta$, while no significant difference was found among these OGD $3 \mathrm{~h}$ groups (Fig. 2B). In addition, NO release from microglia exhibited a progressive increase by 0GD3h following different reoxygenation processes, while OGD3h/R24h reached a peak (Fig. 2C).

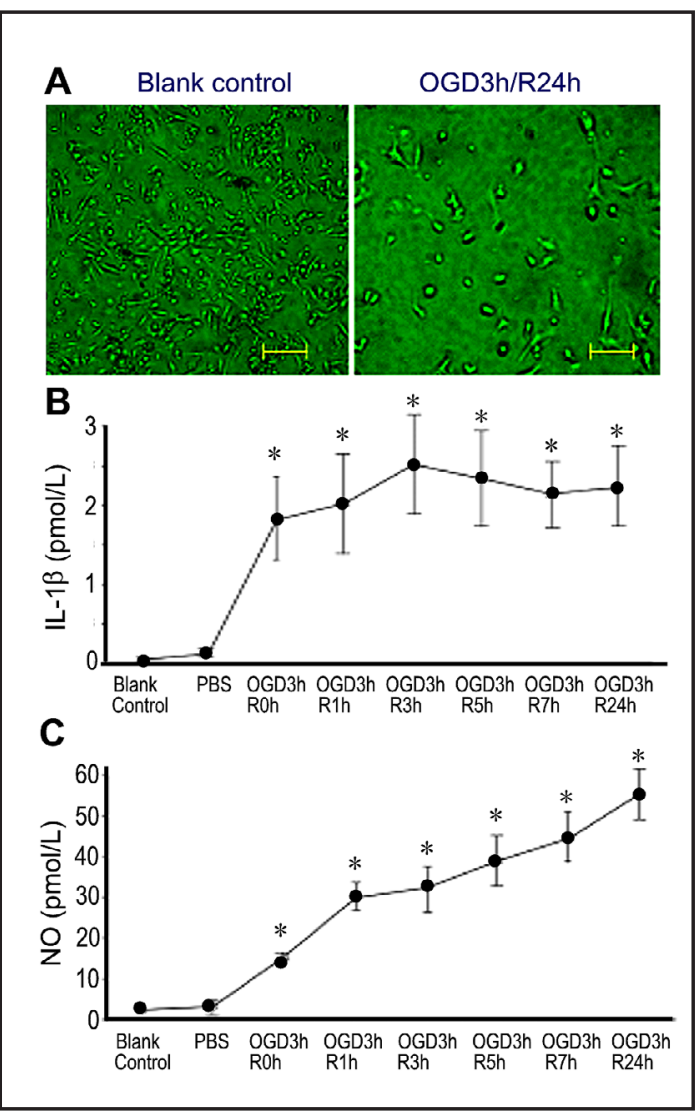

Fig. 2. The neurotoxic factors from microglia induced by $\mathrm{OGD} / \mathrm{R}$ processes. Primary microglia were cultured and treated with OGD3h, followed by different reoxygenation. (A) the morphological variations of microglia at OGD3h/R24h, (B) the level of IL-1 $\beta$ production at different reoxygenation, (C) the level of NO release at different reoxygenation. $*$ means $\mathrm{p}<0.05$; the bar $=10 \mu \mathrm{m}$. 


\section{Cellular Physiology Cell Physiol Biochem 2016;38:1472-1482 and Biochemistry

Fig. 3. NSP-induced inhibition of IL-1 and NO release from microglia treated by OGD/R. Primary microglia were cultured and treated with OGD3h/R24h or OGD1.5h/R24h + NSP. (A) the morphological variations of microglia, (B) the level of IL-1 $\beta$ production, (C) the level of NO release. * means $\mathrm{p}<0.05$ for both OGD/R groups vs. non- OGD/R group and NSP group vs. non-NSP group.; the bar $=10 \mu \mathrm{m}$.

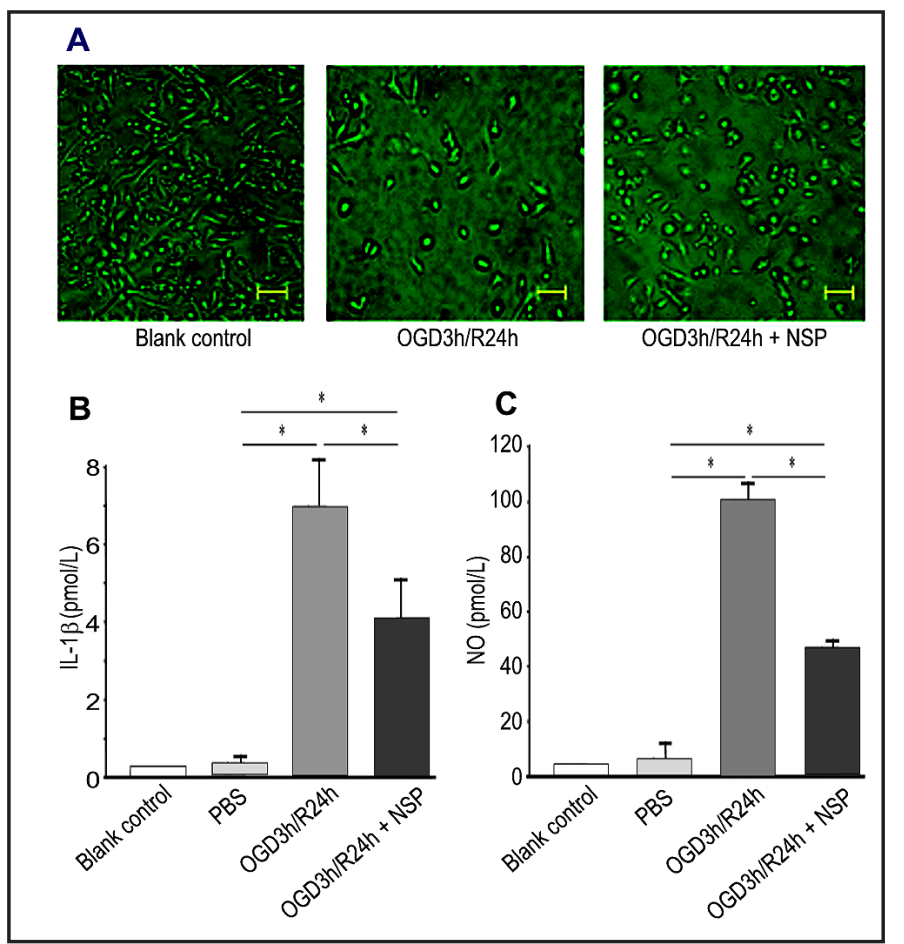

Fig. 4. NSP-induced inhibition of the MAPK pathway on microglia treated by OGD/R. Primary microglia were cultured and treated with OGD3h/R24h or or OGD1.5h/R24h + NSP. Protein extracts were prepared from cultured microglia, and the expression of ERK1/2, p38, and JNK was measured by Western blot. $* \mathrm{p}<0.05$ for both OGD/R groups vs. non- OGD/R group and NSP group vs. non-NSP group.

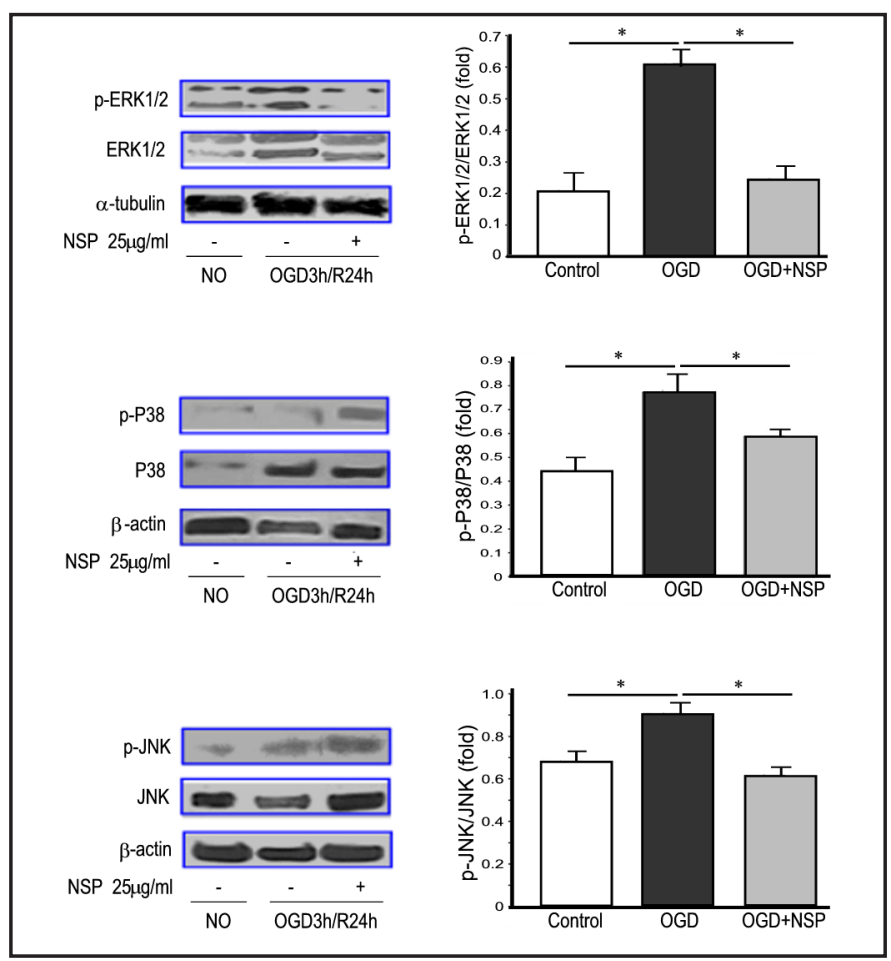

We consider that IL-1 $\beta$ and NO derived from microglia could be related with neuroinflammation and neuron injury in the CNS.

NSP-induced inhibition of $I L-1$ and NO release from microglia treated by $O G D / R$

Figure 3A shows the morphological variations of microglia by OGD 3h/R24h processes and the administration of NSP. The left picture is an image of normal microglia, while the middle column is an image of microglia that had undergone OGD/R processes. The administration of NSP can reduced the loss of microglia induced by OCD/R processes (Fig. 3A). The release of 


\section{Cellular Physiology Cell Physiol Biochem 2016;38:1472-1482 \begin{tabular}{l|l} 
and Biochemistry Published online: April 04, 2016 & $\begin{array}{l}\text { C) 2016 S. Karger AG, Basel } \\
\text { www.karger.com/cpb }\end{array}$ \\
\hline
\end{tabular} \\ Yang et al.: NSP Shows Neuroprotection on Neurons and Microglia-Mediated Inflam- matory Response}

IL-1 $\beta$ from microglia was significantly increased after OGD3h/R24h (Fig. 3B, p<0.05, OGD/R group vs. blank control). However, the administration of NSP significantly decreased IL-1 $\beta$ release (Fig. 3B, p < 0.05). The NO release significantly increased after OGD3h/R24h (Fig. 3C, $\mathrm{p}<0.05, \mathrm{OGD} / \mathrm{R}$ group vs. blank control). However, the administration of NSP significantly decreased NO release (Fig. 3C, $\mathrm{p}<0.05$ ).

NSP-induced inhibition of the MAPK pathway on microglia treated by OGD/R

Western blot analysis was used to assess the activation of the mitogen-activated protein kinase (MAPK) pathway, including the expression of ERK, P38, and JNK. As shown in Fig. 4, OGD3h/R24h processes triggered the upregulation of ERK, p38 and JNK expression ( $p<0.05$ respectively), while the administration of NSP significantly inhibited the expression of ERK, P38, and JNK protein (Fig. 4, p $<0.05$ respectively). Taken together, these results demonstrate that IL-1 $\beta$ and NO derived from microglia may contribute to neuron damage, and that NSP-mediated inhibition of inflammatory molecules should be neuroprotective in the CNS.

\section{Discussion}

The neuroprotective effects of NSP have been widely reported in stroke subjects with complicated and uncertain mechanisms. The aim of the present study is to verify the neuroprotective role of NSP on neurons and microglia-mediated inflammatory response. To the best of our knowledge, it is the first investigation regarding the neuroprotective effects of NSP on neurons and microglia-mediated inflammatory response. The importance of this study corroborates our recently published study on astrocytes [11] and intracerebral hemorrhage mouse models (manuscript submitted), where we have verified the neuroprotective effects of NSP on neurons, microglia-mediated inflammatory response, and astrocytes in vitro, respectively. These findings are beneficial in achieving a deeper insight into the physiological mechanisms of NSP and in contributing to the development or implementation of NSP as a potential neuroprotective agent for treating stroke in the future.

The CNS is certainly the most important part of the nervous system, and comprises neurons and glial cells, including astrocytes $(20 \%-40 \%)$ [28], microglia $(10 \%-15 \%)$ [13], and oligodendrocytes. Glial cells are crucial in maintaining the normal function of the CNS as "supporting cells," which contribute to the supply of nutrients and oxygen to neurons (astrocytes). Furthermore, glial cells insulate, surround, and hold neurons (oligodendrocytes) as well as remove the dead neurons or other foreign bodies directly introduced into the CNS (microglia). For stroke, other than the oligodendrocytes, which provide support and insulation to axons in the CNS, the most important cells are neurons, astrocytes, microglia, and cerebrovascular endothelial cells [29]. In previous studies, we have confirmed the neuroprotective effect of NSP on the cerebrovascular endothelial cells (data not shown). In this study, we observed the neuroprotective effect of NSP on neurons and microglia under the ischemic/hypoxic condition. We found that the OGD3h/R4h processes induced morphological variations of neurons (Fig. 1A), and reduced the rate of neuron survival (Fig. 1B) as well as increased the rate of neuron apoptosis (Fig. 1C) and LDH release (Fig. 1D). The administration of NSP significantly reversed these changes, although this amelioration did not return to the normal level (Fig. 1). Similarly, we found that OGD/R processes also caused morphological changes on microglia (Fig. 2A, 3A), significantly enhancing the release of IL-1 $\beta$ (Fig. 2B, 3B) and NO (Fig. 2C, 3C), whereas these changes were significantly reversed by the administration of NSP (Fig. 3). These results confirmed the neuroprotective effects of NSP directly on neurons or indirectly by microglia-mediated inflammatory response and/or oxidative stress. Considering the previous studies about the role of NSP on astrocytes [11] and cerebrovascular endothelial cells (data not shown), the results in this study demonstrate that NSP may achieve the neuroprotective effect through a variety of resident cells (such as neurons, microglia, astrocytes, oligodendrocytes, vascular endothelial cells and their 


\section{Cellular Physiology Cell Physiol Biochem 2016;38:1472-1482 \begin{tabular}{l|l} 
and Biochemistry Published online: April 04, 2016 & $\begin{array}{l}\text { C) 2016 S. Karger AG, Basel } \\
\text { www.karger.com/cpb }\end{array}$ \\
\hline
\end{tabular} \\ Yang et al.: NSP Shows Neuroprotection on Neurons and Microglia-Mediated Inflam- matory Response}

signaling networks in the brain. These findings are very important in evaluating the worth of NSP as a potential neuroprotective agent for stroke. Because it acts on all the important cells of the CNS affected by stroke, it is possible that NSP can act as a potential neuroprotective agent for stroke. However, we still do not know whether this neuroprotective effect is associated with IPA, and whether NSP shows neuroprotection towards oligodendrocytes, which should be included in the future work of our laboratory.

Another finding seems to indicate that the inhibition of MAPK signaling pathway may play a role in microglia-mediated NSP neuroprotection. It is documented that MAPK signaling pathway is involved in inflammation in various cells [30]. Three families of MAPK pathways, namely ERK, JNK and p38 have been understood [31]. We have known activation any of the three pathways (ERK, JNK and p38MAPK) is harmful, while suppression of any of the three MAPK pathways (ERK, JNK and p38MAPK) can alleviate the injury, is therefore protective $[30,32]$. Our date showed that OGD3h/R24 treatment significantly upregulated expression of all the three pathways, namely ERK, p38, and JNK, whereas the administration of NSP significantly, reversed this upregulation (Fig. 4). These results support that ERK, p38 and JNK are related to the NSP neuroprotection, which should be verified using an antagonist/ agonist of the MAPK pathway in our future study. In comparison with the findings for astrocytes, which indicated that the protective effects of NSP on astrocytes is associated with the NF- $\kappa \mathrm{B}$ signaling pathways other than the MAPK and PI3K/Akt pathways [11], the findings of our study is very interesting. With regard to the neurons, we still do not know which signaling pathways (MAPK, PI3K/Akt or NF-kB?) are involved in the neuroprotection on them. Our future work will detect the related signaling pathways on neurons and will attempt to achieve a better understanding of the signaling pathways involved in the NSP neuroprotection on brain cells.

In the present study, we did not adopt the same methods to detect death of microglia as what we had done on neurons (Fig. 1) and on astrocytes [11] because of the complicated biological characteristics of microglia. We got to know microglia are the resident macrophages in the CNS, which play roles in active immune defense. This means microglia is a double-edged sword including neurotoxicity and neuroprotection [33]. It is difficult to judge it is harmful or beneficial just simply increase or removal microglia itself. We therefore abandoned the experiments of detecting the survival rate or apoptosis rate on microglia. Instead, we found that investigation of the microglia-mediated inflammatory response, namely measurements of the neuroinflammatory molecules like IL-1 $\beta$ and NO may have better clinical significance as to the neuroprotection of NSP concerning microglia.

In conclusion, the present study verified the neuroprotective effects of NSP on neurons and microglia-mediated inflammatory response. Together with our previous investigations on astrocytes and cerebrovascular endothelial cells, we consider that the neuroprotection of NSP acts on several cells in the CNS that are affected by stroke. NSP could be considered as a potential new neuroprotective agent for treating stroke. Another finding in the present study is that NSP neuroprotection on microglia-mediated inflammatory response may be related to the MAPK signaling pathway, which needs to be verified in our future investigations.

\section{Acknowledgements}

LW was supported by a grant from the Science and Technology Commission of Shanghai Municipality (No. 13441902600) and Clinical Medical Research Grant of Chinese medical Association (No. 09010180173). TA was supported by grants from the Japanese Society for the Promotion of Science (Grant-in-Aid for Young Scientists, Type B, No. 20791025 and Grant-in-Aid for Scientific Research C, General, No. 24592157 and 15k10358).

\section{Disclosure Statement}

The authors do not have conflict of interest to declare for this study. 


\section{References}

1 Gelderblom M, Neumann M, Ludewig P, Bernreuther C, Krasemann S, Arunachalam P, Gerloff C, Glatzel M, Magnus T: Deficiency in serine protease inhibitor neuroserpin exacerbates ischemic brain injury by increased postischemic inflammation. PLoS One 2013;8:e63118.

2 Noto R, Santangelo MG, Ricagno S, Mangione MR, Levantino M, Pezzullo M, Martorana V, Cupane A, Bolognesi M, Manno M: The tempered polymerization of human neuroserpin. PLoS One 2012;7:e32444.

3 Hanzel CE, Iulita MF, Eyjolfsdottir H, Hjorth E, Schultzberg M, Eriksdotter M, Cuello AC: Analysis of matrix metallo-proteases and the plasminogen system in mild cognitive impairment and alzheimer's disease cerebrospinal fluid. J Alzheimers Dis 2014;40:667-678.

4 Lasierra-Cirujeda J, Coronel P, Aza M, Gimeno M: Beta-amyloidolysis and glutathione in alzheimer's disease. J Blood Med 2013;4:31-38.

5 Zhang ZH, Yu LJ, Hui XC, Wu ZZ, Yin KL, Yang H, Xu Y: Hydroxy-safflor yellow a attenuates abeta(1)(-)(4) (2)-induced inflammation by modulating the jak2/stat3/nf-kappab pathway. Brain Res 2014;1563:72-80.

6 Rodriguez-Gonzalez R, Millan M, Sobrino T, Miranda E, Brea D, de la Ossa NP, Blanco M, Perez J, Dorado L, Castellanos M, Lomas DA, Moro MA, Davalos A, Castillo J: The natural tissue plasminogen activator inhibitor neuroserpin and acute ischaemic stroke outcome. Thromb Haemost 2011;105:421-429.

7 Liang W, Chuan-Zhen L, Qiang D, Jian Q, Hui-Min R, Bao-Guo X: Reductions in mrna of the neuroprotective agent, neuroserpin, after cerebral ischemia/reperfusion in diabetic rats. Brain Res 2004;1015:175-180.

8 Ma J, Yu D, Tong Y, Mao M: Effect of neuroserpin in a neonatal hypoxic-ischemic injury model ex vivo. Biol Res 2012;45:357-362.

9 Docagne F, Nicole O, Marti HH, MacKenzie ET, Buisson A, Vivien D: Transforming growth factor-beta1 as a regulator of the serpins/t-pa axis in cerebral ischemia. FASEB J 1999;13:1315-1324.

10 Rodriguez-Gonzalez R, Agulla J, Perez-Mato M, Sobrino T, Castillo J: Neuroprotective effect of neuroserpin in rat primary cortical cultures after oxygen and glucose deprivation and tpa. Neurochem Int 2011;58:337343.

11 Wang L, Zhang Y, Asakawa T, Li W, Han S, Li Q, Xiao B, Namba H, Lu C, Dong Q: Neuroprotective effect of neuroserpin in oxygen-glucose deprivation-and reoxygenation-treated rat astrocytes in vitro. PloS one 2015;10:e0123932.

12 Rolls MM, Jegla TJ: Neuronal polarity: An evolutionary perspective. J Exp Biol 2015;218:572-580.

13 Lawson L, Perry V, Gordon S: Turnover of resident microglia in the normal adult mouse brain. Neuroscience 1992;48:405-415.

14 Bushong EA, Martone ME, Jones YZ, Ellisman MH: Protoplasmic astrocytes in ca1 stratum radiatum occupy separate anatomical domains. J Neurosci 2002;22:183-192.

15 Dissing-Olesen L, Ladeby R, Nielsen HH, Toft-Hansen H, Dalmau I, Finsen B: Axonal lesion-induced microglial proliferation and microglial cluster formation in the mouse. Neuroscience 2007;149:112-122.

16 Kennedy SA, van Diepen AC, van den Hurk CM, Coates LC, Lee TW, Ostrovsky LL, Miranda E, Perez J, Davies MJ, Lomas DA: Expression of the serine protease inhibitor neuroserpin in cells of the human myeloid lineage. Thromb Haemost 2007;97:394-399.

17 Munuswamy-Ramanujam G, Dai E, Liu L, Shnabel M, Sun YM, Bartee M, Lomas DA, Lucas AR: Neuroserpin, a thrombolytic serine protease inhibitor (serpin), blocks transplant vasculopathy with associated modification of t-helper cell subsets. Thromb Haemost 2010;103:545.

18 Zheng D, Chen H, Bartee MY, Williams J, Davids JA, Lomas DA, McFadden G, Lucas AR: Myxomaviral antiinflammatory serpin reduces myeloid-derived suppressor cells and human pancreatic cancer cell growth in mice. J Cancer Sci Ther 2013;5:291-299.

19 Brewer GJ, Torricelli JR, Evege EK, Price PJ: Optimized survival of hippocampal neurons in b27supplemented neurobasal, a new serum-free medium combination. J Neurosci Res 1993;35:567-576.

20 Yan J, Huang Y, Lu Y, Chen J, Jiang H: Repeated administration of ketamine can induce hippocampal neurodegeneration and long-term cognitive impairment via the ros/hif- $1 \alpha$ pathway in developing rats. Cell Physiol Biochem 2014;33:1715-1732.

21 Gebicke-Haerter PJ, Bauer J, Schobert A, Northoff H: Lipopolysaccharide-free conditions in primary astrocyte cultures allow growth and isolation of microglial cells. J Neurosci 1989;9:183-194. 


\section{Cellular Physiology Cell Physiol Biochem 2016;38:1472-1482}

and Biochemistry

22 Gouix E, Buisson A, Nieoullon A, Kerkerian-Le Goff L, Tauskela JS, Blondeau N, Had-Aissouni L: Oxygen glucose deprivation-induced astrocyte dysfunction provokes neuronal death through oxidative stress. Pharmacol Res 2014;87:8-17.

23 Zhou X, Gu J, Gu Y, He M, Bi Y, Chen J, Li T: Human umbilical cord-derived mesenchymal stem cells improve learning and memory function in hypoxic-ischemic brain-damaged rats via an il-8-mediated secretion mechanism rather than differentiation pattern induction. Cell Physiol Biochem 2015;35:2383-2401.

24 Tornusciolo D, Schmidt RE, Roth KA: Simultaneous detection of tdt-mediated dutp-biotin nick end-labeling (tunel)-positive cells and multiple immunohistochemical markers in single tissue sections. Biotechniques 1995;19:800-805.

25 Koh JY, Choi DW: Quantitative determination of glutamate mediated cortical neuronal injury in cell culture by lactate dehydrogenase efflux assay. J Neurosci Methods 1987;20:83-90.

26 Tung WH, Lee IT, Hsieh HL, Yang CM: Ev71 induces cox-2 expression via c-src/pdgfr/pi3k/akt/p42/p44 mapk/ap-1 and nf-kappab in rat brain astrocytes. J Cell Physiol 2010;224:376-386.

27 Wei R, Zhang R, Xie Y, Shen L, Chen F: Hydrogen suppresses hypoxia/reoxygenation-induced cell death in hippocampal neurons through reducing oxidative stress. Cell Physiol Biochem 2015;36:585-598.

28 Verkhratsky A, Butt AM: „Numbers: How many glial cells are in the brain?“; Glial physiology and pathophysiology. Chichester, John Wiley \& Sons, 2013, pp 93-96.

29 Takano T, Oberheim N, Cotrina ML, Nedergaard M: Astrocytes and ischemic injury. Stroke 2009;40:S8-S12.

30 Su D, Ma J, Zhang Z, Tian Y, Shen B: Protective effects of ucf-101 on cerebral ischemia-reperfusion (cir) is depended on the mapk/p38/erk signaling pathway. Cell Mol Neurobiol DOI:10.1007/s10571-015-0275-6.

31 Johnson GL, Lapadat R: Mitogen-activated protein kinase pathways mediated by erk, jnk, and p38 protein kinases. Science 2002;298:1911-1912.

32 Goldsmith CS, Bell-Pedersen D: Diverse roles for mapk signaling in circadian clocks. Adv Genet 2013;84:139.

33 Waisman A, Liblau RS, Becher B: Innate and adaptive immune responses in the cns. Lancet Neurol 2015;14:945-955. 